\title{
On the Existence of Ground State Solutions of the Periodic Discrete Coupled Nonlinear Schrödinger Lattice
}

\author{
Meihua Huang ${ }^{1,2}$ and Zhan Zhou ${ }^{1,2}$ \\ ${ }^{1}$ School of Mathematics and Information Science, Guangzhou University, Guangzhou, Guangdong 510006, China \\ ${ }^{2}$ Key Laboratory of Mathematics and Interdisciplinary Sciences of Guangdong Higher Education Institutes, Guangzhou University, \\ Guangzhou, Guangdong 510006, China
}

Correspondence should be addressed to Zhan Zhou; zzhou0321@hotmail.com

Received 1 July 2013; Accepted 6 September 2013

Academic Editor: Bo-Qing Dong

Copyright (C) 2013 M. Huang and Z. Zhou. This is an open access article distributed under the Creative Commons Attribution License, which permits unrestricted use, distribution, and reproduction in any medium, provided the original work is properly cited.

We study the existence of ground state solutions of the periodic discrete coupled nonlinear Schrödinger lattice by using the Nehari manifold approach combined with periodic approximations. We show that both of the components of the ground state solutions are not zero.

\section{Introduction}

In this paper, we consider the coupled discrete Schrödinger system

$$
\begin{aligned}
& i \frac{d u_{n}}{d t}=-(\mathscr{A} u)_{n}+b_{1 n} u_{n}-a_{1}\left|u_{n}\right|^{2} u_{n}-a_{3}\left|v_{n}\right|^{2} u_{n}, \\
& i \frac{d v_{n}}{d t}=-(\mathscr{A} v)_{n}+b_{2 n} v_{n}-a_{2}\left|v_{n}\right|^{2} v_{n}-a_{3}\left|u_{n}\right|^{2} v_{n},
\end{aligned}
$$

where $a_{i}$ is a positive constant, $\left\{b_{j n}\right\}$ is a real valued $T$-periodic sequence, $i=1,2,3$, and $j=1,2 . \mathscr{A}$ is the discrete Laplacian operator defined as $(\mathscr{A} u)_{n}=u_{n+1}+u_{n-1}-2 u_{n}$.

System (1) could be viewed as the discretization of the two-component system of time-dependent nonlinear GrossPitaevskii system (see [1] for more detail) as

$$
\begin{aligned}
i \hbar \partial_{t} u & =-\frac{\hbar^{2}}{2 m} \Delta u+b_{1}(x) u-a_{1}|u|^{2} u-a_{3}|v|^{2} u, \\
i \hbar \partial_{t} v & =-\frac{\hbar^{2}}{2 m} \Delta v+b_{2}(x) v-a_{2}|v|^{2} v-a_{3}|u|^{2} v .
\end{aligned}
$$

It is well known that coupled nonlinear Schrödinger equations arise quite naturally in nonlinear optics [2] and
Bose-Einstein condensates. Bose-Einstein condensation for a mixture of different interaction atomic species with the same mass was realized in 1997 (see [3]), which stimulated various analytical and numerical results on the ground state solutions of system (2). The discrete nonlinear Schrödinger equations (DNLS) have a crucial role in the modeling of a great variety of phenomena, ranging from solid-state and condensed-matter physics to biology. During the last years, there has been a growing interest in approaches to the existence problem for ground states. We refer to the continuation methods in $[4,5]$, which have been proved to be powerful for both theoretical considerations and numerical computations (see [6]), to [7], which exploits spatial dynamics and centre manifold reduction, to the variational methods in [8-15], which rely on critical point techniques (linking theorems, the Nehari manifold), and to the Krasnoselskii fixed point theorem together with a suitable compactness criterion [16].

The aim of this paper is to study the discrete solitons of (1), that is, solutions of the form

$$
\begin{array}{r}
u_{n}=\exp \left(-i \omega_{1} t\right) \phi_{n}, \quad v_{n}=\exp \left(-i \omega_{2} t\right) \psi_{n}, \quad n \in \mathbb{Z}, \\
\lim _{|n| \rightarrow \infty} u_{n}=0, \quad \lim _{|n| \rightarrow \infty} v_{n}=0,
\end{array}
$$


where the amplitudes $\phi_{n}$ and $\psi_{n}$ are supposed to be real. Inserting the ansatz of the discrete solitons (3) into (1), we obtain the following equivalent algebraic equations:

$$
\begin{aligned}
& -(\mathscr{A} \phi)_{n}-\omega_{1} \phi_{n}+b_{1 n} \phi_{n}-a_{1}\left|\phi_{n}\right|^{2} \phi_{n}-a_{3}\left|\psi_{n}\right|^{2} \phi_{n}=0, \\
& -(\mathscr{A} \psi)_{n}-\omega_{2} \psi_{n}+b_{2 n} \psi_{n}-a_{2}\left|\psi_{n}\right|^{2} \psi_{n}-a_{3}\left|\phi_{n}\right|^{2} \psi_{n}=0,
\end{aligned}
$$

and (4) becomes

$$
\lim _{|n| \rightarrow \infty} \phi_{n}=0, \quad \lim _{|n| \rightarrow \infty} \psi_{n}=0 .
$$

In fact, we consider the following more general equations:

$$
\begin{aligned}
& \left(L_{1} \phi\right)_{n}-\omega_{1} \phi_{n}-a_{1}\left|\phi_{n}\right|^{2} \phi_{n}-a_{3}\left|\psi_{n}\right|^{2} \phi_{n}=0, \\
& \left(L_{2} \psi\right)_{n}-\omega_{2} \psi_{n}-a_{2}\left|\psi_{n}\right|^{2} \psi_{n}-a_{3}\left|\phi_{n}\right|^{2} \psi_{n}=0,
\end{aligned}
$$

where $L_{1}$ and $L_{2}$ are the second-order difference operators defined by

$$
\begin{aligned}
& L_{1} \phi_{n}=\alpha_{n} \phi_{n+1}+\alpha_{n-1} \phi_{n-1}+\beta_{n} \phi_{n}, \\
& L_{2} \psi_{n}=\gamma_{n} \psi_{n+1}+\gamma_{n-1} \psi_{n-1}+\delta_{n} \psi_{n},
\end{aligned}
$$

where $\left\{\alpha_{n}\right\},\left\{\beta_{n}\right\},\left\{\gamma_{n}\right\}$, and $\left\{\delta_{n}\right\}$ are real valued $T$-periodic sequences, and $a_{1}, a_{2}$, and $a_{3}$ are positive numbers. Obviously, (5) is a special case of (7) with $\alpha_{n} \equiv-1, \beta_{n} \equiv 2+b_{1 n}, \gamma_{n} \equiv-1$, and $\delta_{n} \equiv 2+b_{2 n}$.

Since, for $i=1,2$, the operator $L_{i}$ is a bounded and selfadjoint operator in $l^{2}$, its spectrum $\sigma\left(L_{i}\right)$ has a band structure; that is, $\sigma\left(L_{i}\right)$ is a union of a finite number of closed intervals [17]. The complement $\mathbb{R}-\sigma\left(L_{i}\right)$ consists of a finite number of open intervals called spectral gaps and two of them are semi-infinite which are denoted by $\left(-\infty, \theta_{1 i}\right)$ and $\left(\theta_{2 i}, \infty\right)$, respectively.

In this paper, we consider two types of solutions to (7) as follows: (i) $2 k T$-periodic, that is, $\phi_{n+2 k T}=\phi_{n}, \psi_{n+2 k T}=$ $\psi_{n}$, and (ii) discrete solitons. Actually, in case (ii), we look for solutions in the space $l^{2} \times l^{2}=l^{2}(\mathbb{Z}) \times l^{2}(\mathbb{Z})$; then (6) holds naturally. System (7) has a trivial solution $\phi_{n} \equiv 0, \psi_{n} \equiv 0$. We are looking for nontrivial solutions.

The main idea in this paper is as follows. First, we consider (7) in a finite $2 k T$-periodic sequence space, and $\omega_{i}$ is not a spectrum of the corresponding operator $L_{i}, i=1,2$. By using the Nehari manifold approach, we obtain the existence of $2 k T$-periodic solutions. Then we show that these solutions have upper and lower bounds. Finally, by an approximation technique, we prove that the limit of these solutions exists and is the solution of (7) in $l^{2} \times l^{2}$. Compared with the existence of ground state solutions of the DNLS, the difficulty is that we need to show that both of the components of the ground state solutions are not zero.

The remaining of this paper is organized as follows. First, in Section 2, we establish the variational framework associated with (7) and introduce the Nehari manifolds. Then, in Section 3, we present a sufficient condition on the existence of $2 k T$-periodic solutions and nontrivial solutions in $l^{2} \times l^{2}$ of (7).

\section{Preliminaries and the Nehari Manifold}

In this section, we first establish the variational framework associated with (7).

Let $S$ be the set of the following form:

$$
S=\left\{\phi=\left\{\phi_{n}\right\}: \phi_{n} \in \mathbb{R}, n \in \mathbb{Z}\right\}
$$

For any fixed positive integer $k$, we define the subspace $X_{k}$ of $S$ as

$$
X_{k}=\left\{\phi \in S: \phi_{n+2 k T}=\phi_{n}, n \in \mathbb{Z}\right\}
$$

Obviously, $X_{k}$ is isomorphic to $R^{2 k T}$ and hence $X_{k}$ can be equipped with the inner product $(\cdot, \cdot)_{k}$ and norm $\|\cdot\|_{k}$ as

$$
\begin{gathered}
(\phi, \psi)_{k}=\sum_{n=-k T}^{k T-1} \phi_{n} \psi_{n}, \quad \phi, \psi \in X_{k}, \\
\|\phi\|_{k}=\left(\sum_{n=-k T}^{k T-1} \phi_{n}^{2}\right)^{1 / 2}, \quad \phi \in X_{k},
\end{gathered}
$$

respectively. Sometimes, we will consider $l^{p}$ norm on $X_{k}$ as

$$
\|\phi\|_{k p}=\left(\sum_{n=-k T}^{k T-1}\left|\phi_{n}\right|^{p}\right)^{1 / p}, \quad 1 \leq p<\infty .
$$

We also define a norm $\|\cdot\|_{k \infty}$ on $X_{k}$ by

$$
\|\phi\|_{k \infty}=\max \left\{\left|\phi_{n}\right|:-k T \leq n \leq k T-1\right\}, \quad \phi \in X_{k} .
$$

The symbol $X$ stands for the space $X=l^{2}$ with the norm

$$
\|\phi\|=\left(\sum_{n \in \mathbb{Z}} \phi_{n}^{2}\right)^{1 / 2}, \quad \phi \in X
$$

and the inner product

$$
(\phi, \psi)=\sum_{n \in \mathbb{Z}} \phi_{n} \psi_{n}, \quad \phi, \psi \in X
$$

We also consider $l^{p}$ norm on $X$ as

$$
\begin{gathered}
\|\phi\|_{p}=\left(\sum_{n \in \mathbb{Z}}\left|\phi_{n}\right|^{p}\right)^{1 / p}, \quad 1 \leq p<\infty, \\
\|\phi\|_{\infty}=\max \left\{\left|\phi_{n}\right|: n \in \mathbb{Z}\right\} .
\end{gathered}
$$

We mention that

$$
\|\phi\|_{k p} \leq\|\phi\|_{k q}, \quad\|\phi\|_{p} \leq\|\phi\|_{q}
$$

where $1 \leq q \leq p \leq \infty$. 
Consider the functionals $J_{k}$ on $X_{k} \times X_{k}$ and $J$ on $X \times X$ defined by

$$
\begin{aligned}
J_{k}(\phi, \psi)= & \frac{1}{2}\left(\left(L_{1}-\omega_{1}\right) \phi, \phi\right)_{k}+\frac{1}{2}\left(\left(L_{2}-\omega_{2}\right) \psi, \psi\right)_{k} \\
& -\frac{1}{4} \sum_{n=-k T}^{k T-1}\left(a_{1} \phi_{n}^{4}+a_{2} \psi_{n}^{4}+2 a_{3} \phi_{n}^{2} \psi_{n}^{2}\right), \\
J(\phi, \psi)= & \frac{1}{2}\left(\left(L_{1}-\omega_{1}\right) \phi, \phi\right)+\frac{1}{2}\left(\left(L_{2}-\omega_{2}\right) \psi, \psi\right) \\
& -\frac{1}{4} \sum_{n \in \mathbb{Z}}\left(a_{1} \phi_{n}^{4}+a_{2} \psi_{n}^{4}+2 a_{3} \phi_{n}^{2} \psi_{n}^{2}\right),
\end{aligned}
$$

respectively. Then $J_{k}(\phi, \psi) \in C^{1}\left(X_{k} \times X_{k}, \mathbb{R}\right)$ and its derivative is given by

$$
\begin{aligned}
\left(J_{k}^{\prime}(\phi, \psi),(\phi, \psi)\right)= & \left(\left(L_{1}-\omega_{1}\right) \phi, \phi\right)_{k}+\left(\left(L_{2}-\omega_{2}\right) \psi, \psi\right)_{k} \\
& -\sum_{n=-k T}^{k T-1}\left(a_{1} \phi_{n}^{4}+a_{2} \psi_{n}^{4}+2 a_{3} \phi_{n}^{2} \psi_{n}^{2}\right),
\end{aligned}
$$

and $J(\phi, \psi) \in C^{1}(X \times X, \mathbb{R})$ and its derivative is given by

$$
\begin{aligned}
\left(J^{\prime}(\phi, \psi),(\phi, \psi)\right)= & \left(\left(L_{1}-\omega_{1}\right) \phi, \phi\right)+\left(\left(L_{2}-\omega_{2}\right) \psi, \psi\right) \\
& -\sum_{n \in \mathbb{Z}}\left(a_{1} \phi_{n}^{4}+a_{2} \psi_{n}^{4}+2 a_{3} \phi_{n}^{2} \psi_{n}^{2}\right) .
\end{aligned}
$$
is,

Let $\zeta_{i}$ be the distance from $\omega_{i}$ to the spectrum $\sigma\left(L_{i}\right)$; that

$$
\zeta_{i}=\theta_{1 i}-\omega_{i}, \quad i=1,2 .
$$

Let $\zeta=\min \left\{\zeta_{1}, \zeta_{2}\right\}$. Then, we have

$$
\begin{array}{r}
\left(\left(L_{i}-\omega_{i}\right) \phi, \phi\right)_{k} \geq \zeta\|\phi\|_{k}^{2}, \quad \phi \in X_{k}, \quad i=1,2, \\
\left(\left(L_{i}-\omega_{i}\right) \phi, \phi\right) \geq \zeta\|\phi\|^{2}, \quad \phi \in X, \quad i=1,2 .
\end{array}
$$

Furthermore, we let

$$
\begin{aligned}
& \lambda_{1 *}=\min _{n \in \mathbb{Z}}\left\{\left|\beta_{n}-\omega_{1}\right|-\left|\alpha_{n-1}\right|-\left|\alpha_{n}\right|\right\}, \\
& \lambda_{1}^{*}=\max _{n \in \mathbb{Z}}\left\{\left|\beta_{n}-\omega_{1}\right|+\left|\alpha_{n-1}\right|+\left|\alpha_{n}\right|\right\}, \\
& \lambda_{2 *}=\min _{n \in \mathbb{Z}}\left\{\left|\delta_{n}-\omega_{2}\right|-\left|\gamma_{n-1}\right|-\left|\gamma_{n}\right|\right\}, \\
& \lambda_{2}^{*}=\max _{n \in \mathbb{Z}}\left\{\left|\delta_{n}-\omega_{2}\right|+\left|\gamma_{n-1}\right|+\left|\gamma_{n}\right|\right\} .
\end{aligned}
$$

Then, obviously, $\lambda_{1}^{*}>0$ and $\lambda_{2}^{*}>0$.

Denote

$$
\lambda=\max _{n \in \mathbb{Z}}\left\{\left|\alpha_{n}-\gamma_{n}\right|+\left|\alpha_{n-1}-\gamma_{n-1}\right|+\left|\beta_{n}-\delta_{n}\right|\right\} .
$$

Then

$$
\left\|\left(L_{1}-L_{2}\right) \phi\right\| \leq \lambda\|\phi\|, \quad \phi \in X .
$$

Next, we study the main properties of the Nehari manifolds with the functionals $J_{k}$ and $J$.

Let

$$
\begin{array}{cc}
I_{k}(\phi, \psi)=\left(J_{k}^{\prime}(\phi, \psi),(\phi, \psi)\right), & (\phi, \psi) \in X_{k} \times X_{k}, \\
I(\phi, \psi)=\left(J^{\prime}(\phi, \psi),(\phi, \psi)\right), & (\phi, \psi) \in X \times X .
\end{array}
$$

Then $I_{k}$ and $I$ are $C^{1}$ functionals and their derivatives are given by

$$
\begin{aligned}
\left(I_{k}^{\prime}(\phi, \psi),(\phi, \psi)\right)= & 2\left(\left(L_{1}-\omega_{1}\right) \phi, \phi\right)_{k}+2\left(\left(L_{2}-\omega_{2}\right) \psi, \psi\right)_{k} \\
& -4 \sum_{n=-k T}^{k T-1}\left(a_{1} \phi_{n}^{4}+a_{2} \psi_{n}^{4}+2 a_{3} \phi_{n}^{2} \psi_{n}^{2}\right), \\
\left(I^{\prime}(\phi, \psi),(\phi, \psi)\right)= & 2\left(\left(L_{1}-\omega_{1}\right) \phi, \phi\right)+2\left(\left(L_{2}-\omega_{2}\right) \psi, \psi\right) \\
& -4 \sum_{n \in \mathbb{Z}}\left(a_{1} \phi_{n}^{4}+a_{2} \psi_{n}^{4}+2 a_{3} \phi_{n}^{2} \psi_{n}^{2}\right),
\end{aligned}
$$

respectively. The Nehari manifolds are defined as follows:

$$
\begin{aligned}
N_{k} & =\left\{(\phi, \psi) \in X_{k} \times X_{k}: I_{k}(\phi, \psi)=0,(\phi, \psi) \neq 0\right\}, \\
N & =\{(\phi, \psi) \in X \times X: I(\phi, \psi)=0,(\phi, \psi) \neq 0\} .
\end{aligned}
$$

Note that $N$ contains all critical points of $J$ in $X \times X$.

To prove the main results, we need some lemmas on the Nehari manifolds.

Lemma 1. Assume that $\omega_{1}<\theta_{11}$ and $\omega_{2}<\theta_{12}$ hold. Then the sets $N_{k}$ and $N$ are nonempty closed $C^{1}$ submanifolds in $X_{k} \times X_{k}$ and $X \times X$, respectively. The derivatives $I_{k}^{\prime}$ and $I^{\prime}$ are nonzero on the corresponding Nehari manifolds. Moreover, there exists $\eta>0$ such that $\|\phi\|_{k}^{2}+\|\psi\|_{k}^{2} \geq \eta,(\phi, \psi) \in N_{k}$ and $\|\phi\|^{2}+\|\psi\|^{2} \geq$ $\eta,(\phi, \psi) \in N$.

Proof. The proofs for both cases are similar. We only provide the proof for the case of $N_{k}$ as an illustration.

First, we show that $N_{k} \neq \emptyset$.

Let $(\phi, \psi) \in X_{k} \times X_{k}-\{(0,0)\}$. Then by (21)

$$
\begin{array}{r}
I_{k}(t \phi, t \psi)=t^{2}\left(\left(\left(L_{1}-\omega_{1}\right) \phi, \phi\right)_{k}+\left(\left(L_{2}-\omega_{2}\right) \psi, \psi\right)_{k}\right. \\
\left.-t^{2} \sum_{n=-k T}^{k T-1}\left(a_{1} \phi_{n}^{4}+a_{2} \psi_{n}^{4}+2 a_{3} \phi_{n}^{2} \psi_{n}^{2}\right)\right) .
\end{array}
$$

Noticing that $\left(\left(L_{1}-\omega_{1}\right) \phi, \phi\right)_{k}>0$ and $\left(\left(L_{2}-\omega_{2}\right) \psi, \psi\right)_{k}>0$, by (33), we see that $I_{k}(t \phi, t \psi)>0$ for $t>0$ small enough and $I_{k}(t \phi, t \psi)<0$ for $t>0$ large enough. As a consequence, there exists $t_{0}>0$ such that $I_{k}\left(t_{0} \phi, t_{0} \psi\right)=0$; that is, $\left(t_{0} \phi, t_{0} \psi\right) \in$ $N_{k}$. 

have

Let $(\phi, \psi) \in N_{k}$. By (21), (30), and the definition of $N_{k}$, we

$$
\begin{aligned}
\left(I_{k}^{\prime}(\phi, \psi),(\phi, \psi)\right) & =\left(I_{k}^{\prime}(\phi, \psi),(\phi, \psi)\right)-2 I_{k}(\phi, \psi) \\
& =-2 \sum_{n=-k T}^{k T-1}\left(a_{1} \phi_{n}^{4}+a_{2} \psi_{n}^{4}+2 a_{3} \phi_{n}^{2} \psi_{n}^{2}\right)<0 .
\end{aligned}
$$

Hence, $I_{k}^{\prime} \neq 0$, and the implicit function theorem implies that $N_{k}$ is a $C^{1}$ submonifold in $X_{k} \times X_{k}$.

Now let us prove the last statement of the lemma. Let $(\phi, \psi) \in N_{k}$. By the assumption of Lemma 1 and the definition of $N_{k}$, we have

$$
\begin{aligned}
& \left(\left(L_{1}-\omega_{1}\right) \phi, \phi\right)_{k}+\left(\left(L_{2}-\omega_{2}\right) \psi, \psi\right)_{k} \\
& \quad=\sum_{n=-k T}^{k T-1}\left(a_{1} \phi_{n}^{4}+a_{2} \psi_{n}^{4}+2 a_{3} \phi_{n}^{2} \psi_{n}^{2}\right) \\
& \leq a^{*}\left(\|\phi\|_{k 4}^{2}+\|\psi\|_{k 4}^{2}\right)^{2} \leq a^{*}\left(\|\phi\|_{k}^{2}+\|\psi\|_{k}^{2}\right)^{2},
\end{aligned}
$$

where $a^{*}=\max \left\{a_{1}, a_{2}, a_{3}\right\}$. Let $\eta=\zeta / a^{*}>0$. Then, by (24) and (35), it is easy to see that

$$
\zeta\left(\|\phi\|_{k}^{2}+\|\psi\|_{k}^{2}\right) \leq a^{*}\left(\|\phi\|_{k}^{2}+\|\psi\|_{k}^{2}\right)^{2},
$$

which implies that

$$
\|\phi\|_{k}^{2}+\|\psi\|_{k}^{2} \geq \frac{\zeta}{a^{*}}=\eta .
$$

Closedness of $N_{k}$ is obvious. The proof is completed.

Lemma 2. Assume that $\omega_{1}<\theta_{11}$ and $\omega_{2}<\theta_{12}$ hold. Then there exists $\rho>0$ such that $J_{k}(\phi, \psi) \geq \rho$ for all $(\phi, \psi) \in N_{k}$.

Proof. For any $(\phi, \psi) \in N_{k}$, we have

$$
\begin{aligned}
J_{k}(\phi, \psi) & =J_{k}(\phi, \psi)-\frac{1}{4} I_{k}(\phi, \psi) \\
& =\frac{1}{4}\left(\left(\left(L_{1}-\omega_{1}\right) \phi, \phi\right)_{k}+\left(\left(L_{2}-\omega_{2}\right) \psi, \psi\right)_{k}\right) \\
& \geq \frac{\zeta}{4}\left(\|\phi\|_{k}^{2}+\|\psi\|_{k}^{2}\right) .
\end{aligned}
$$

By Lemma 1, we know that $\|\phi\|_{k}^{2}+\|\psi\|_{k}^{2} \geq \eta$. Hence, let $\rho=$ $(\zeta \eta) / 4>0$. Then (38) implies that $J_{k}(\phi, \psi) \geq \rho$. The proof is completed.

Lemma 3. For $(\phi, \psi) \in N_{k}$, the function $J_{k}(t \phi, t \psi), t>0$, has a unique critical point at $t=1$, which is, actually, a global maximum. The same statement holds for $N$ and $J$.

Proof. Let $F(t)=J_{k}(t \phi, t \psi), t>0,(\phi, \psi) \in N_{k}$. Computing the derivative of $F$, we have

$$
F^{\prime}(t)=t\left(1-t^{2}\right) \sum_{n=-k T}^{k T-1}\left(a_{1} \phi_{n}^{4}+a_{2} \psi_{n}^{4}+2 a_{3} \phi_{n}^{2} \psi_{n}^{2}\right) .
$$

This shows that $t=1$ is a unique maximum point. The proof is completed.
Lemma 4. Let $(\bar{\phi}, \bar{\psi})$ be a minimizer of the functional $J_{k}(\phi, \psi)$ constrained on the Nehari manifold $N_{k}$; that is,

$$
J_{k}(\bar{\phi}, \bar{\psi})=m_{k}=\inf _{(\phi, \psi) \in N} J_{k}(\phi, \psi),
$$

and then $(\bar{\phi}, \bar{\psi})$ is a nontrivial $2 k T$-periodic solution to (7), which is called a nontrivial periodic ground state solution to (7).

Proof. According to Lagrange multiplier method, $(\bar{\phi}, \bar{\psi})$ is the critical point of the functional $J_{k}(\phi, \psi)+\Lambda I_{k}(\phi, \psi)$. Thus $I_{k}(\bar{\phi}, \bar{\psi})=0$, and for arbitrary $(\phi, \psi) \in X_{k} \times X_{k}$,

$$
\left(J_{k}^{\prime}(\bar{\phi}, \bar{\psi})+\Lambda I_{k}^{\prime}(\bar{\phi}, \bar{\psi}),(\phi, \psi)\right)=0 .
$$

After taking $(\phi, \psi)=(\bar{\phi}, \bar{\psi})$, we obtain

$$
\Lambda\left(I_{k}^{\prime}(\bar{\phi}, \bar{\psi}),(\bar{\phi}, \bar{\psi})\right)=0 .
$$

But

$$
\begin{aligned}
& \left(I_{k}^{\prime}(\bar{\phi}, \bar{\psi}),(\bar{\phi}, \bar{\psi})\right) \\
& =2\left(\left(L_{1}-\omega_{1}\right) \bar{\phi}, \bar{\phi}\right)_{k}+2\left(\left(L_{2}-\omega_{2}\right) \bar{\psi}, \bar{\psi}\right)_{k} \\
& \quad-4 \sum_{n=-k T}^{k T-1}\left(a_{1}\left(\bar{\phi}_{n}\right)^{4}+a_{2}\left(\bar{\psi}_{n}\right)^{4}+2 a_{3}\left(\bar{\phi}_{n}\right)^{2}\left(\bar{\psi}_{n}\right)^{2}\right) \\
& =-2 \sum_{n=-k T}^{k T-1}\left(a_{1}\left(\bar{\phi}_{n}\right)^{4}+a_{2}\left(\bar{\psi}_{n}\right)^{4}+2 a_{3}\left(\bar{\phi}_{n}\right)^{2}\left(\bar{\psi}_{n}\right)^{2}\right)<0 .
\end{aligned}
$$

Thus, $\Lambda=0$ and

$$
\left(J_{k}^{\prime}(\bar{\phi}, \bar{\psi}),(\phi, \psi)\right)=0,
$$

for any $(\phi, \psi) \in X_{k} \times X_{k}$. Take $(\phi, \psi)=\left(e^{(j)}, 0\right)$ and $(\phi, \psi)=$ $\left(0, e^{(j)}\right)$ in (44) for $j \in \mathbb{Z} \cap[-k T, k T-1]$, where

$$
e_{n}^{(j)}= \begin{cases}1, & n=j, \\ 0, & n \neq j .\end{cases}
$$

We see that $J_{k}^{\prime}(\bar{\phi}, \bar{\psi})=0$. Thus, $(\bar{\phi}, \bar{\psi})$ is a nontrivial $2 k T$ periodic ground state solution to (7). The proof is completed.

Through a similar argument to the proof of Lemma 4, we get the following lemma.

Lemma 5. Let $(\bar{\phi}, \bar{\psi})$ be a minimizer of the functional $J(\phi, \psi)$ constrained on the Nehari manifold $N$; that is,

$$
J(\bar{\phi}, \bar{\psi})=m=\inf _{(\phi, \psi) \in N} J_{k}(\phi, \psi),
$$

and then $(\bar{\phi}, \bar{\psi})$ is a nontrivial discrete soliton to (7), which is called a nontrivial ground state solution to (7). 


\section{Main Results}

In this section, we will establish some sufficient conditions on the existence of $2 k T$-periodic solutions and nontrivial solutions in $X \times X$ of (7).

We start with the following.

Lemma 6. Assume that $\omega_{1}<\theta_{11}$ and $\omega_{2}<\theta_{12}$ hold. Then the minimum value in (40) is attained.

Proof. Let $\left\{\left(\phi^{(j)}, \psi^{(j)}\right)\right\} \subset N_{k}$ be a minimizing sequence for $J_{k}$; that is, $J_{k}\left(\phi^{(j)}, \psi^{(j)}\right) \rightarrow m_{k}$ as $j \rightarrow+\infty$. The fact that

$$
\begin{array}{r}
J_{k}\left(\phi^{(j)}, \psi^{(j)}\right)=J_{k}\left(\phi^{(j)}, \psi^{(j)}\right)-\frac{1}{2} I_{k}\left(\phi^{(j)}, \psi^{(j)}\right) \\
=\frac{1}{4} \sum_{n=-k T}^{k T-1}\left(a_{1}\left(\phi_{n}^{(j)}\right)^{4}+a_{2}\left(\psi_{n}^{(j)}\right)^{4}\right. \\
\left.+2 a_{3}\left(\phi_{n}^{(j)}\right)^{2}\left(\psi_{n}^{(j)}\right)^{2}\right)
\end{array}
$$

shows that $\left\|\left(\phi^{(j)}, \psi^{(j)}\right)\right\|_{k \infty}$ is bounded. Since the space $X_{k} \times$ $X_{k}$ is finite dimensional, so the norm $\|(\cdot, \cdot)\|_{k \infty}$ is equivalent to the Euclidean norm on $X_{k} \times X_{k}$, and the sequence $\left\{\left(\phi^{(j)}, \psi^{(j)}\right)\right\}$ is bounded. Passing to a subsequence, we can assume that $\left(\phi^{(j)}, \psi^{(j)}\right)$ converges to $(\phi, \psi) \in X_{k} \times X_{k}$. Since the set $N_{k}$ is closed and the functional $J_{k}$ is continuous, we obtain that $(\phi, \psi) \in N_{k}$ and $J_{k}(\phi, \psi)=m_{k}$. The proof is completed.

To obtain a nontrivial solutions in $X \times X$ of (7), we need the following lemma.

Lemma 7. Assume that $\omega_{1}<\theta_{11}$ and $\omega_{2}<\theta_{12}$ hold. Let $\left(\phi^{(k)}\right.$, $\left.\psi^{(k)}\right)$ be a $2 k T$-periodic ground state solution, that is, a solution of (40). Then the sequences $\left\{m_{k}\right\}$ and $\left\{\left\|\phi^{(k)}\right\|_{k}^{2}+\left\|\psi^{(k)}\right\|_{k}^{2}\right\}$ are bounded above and below away from zero.

Proof. By Lemma 2, $\left\{m_{k}\right\}$ is obviously bounded below away from zero. Let

$$
\left(\widetilde{\phi}_{n}^{(k)}, \widetilde{\psi}_{n}^{(k)}\right)= \begin{cases}(1,0), & n=0, \\ (0,0), & n \in \mathbb{Z} \cap[-k T, k T-1]-\{0\},\end{cases}
$$

so $\left(\widetilde{\phi}^{(k)}, \widetilde{\psi}^{(k)}\right) \in X_{k} \times X_{k}-\{(0,0)\}$, as in the beginning of the proof of Lemma 1; there exists $t_{k}=\sqrt{\left(\beta_{0}-\omega_{1}\right) / a_{1}}$ such that $I_{k}\left(t_{k} \widetilde{\phi}^{(k)}, t_{k} \widetilde{\psi}^{(k)}\right)=0$; that is, $\left(t_{k} \widetilde{\phi}^{(k)}, t_{k} \widetilde{\psi}^{(k)}\right) \in N_{k}$. Therefore, $\left(\beta_{0}-\omega_{1}\right)^{2} /\left(4 a_{1}\right)=J_{k}\left(t_{k} \widetilde{\phi}^{(k)}, t_{k} \widetilde{\psi}^{(k)}\right) \geq m_{k}$. And $m_{k}=$ $J_{k}\left(\phi^{(k)}, \psi^{(k)}\right)$ is bounded above.

Next, we prove that $\left\{\left\|\phi^{(k)}\right\|_{k}^{2}+\left\|\psi^{(k)}\right\|_{k}^{2}\right\}$ are bounded above and below away from zero. By the previous proof, we see that $m_{k}=J_{k}\left(\phi^{(k)}, \psi^{(k)}\right)$ is bounded above and below away from zero; that is, there exist $\rho>0$ and $\varrho>0$ such that $\rho \leq J_{k}\left(\phi^{(k)}, \psi^{(k)}\right) \leq \varrho$. Then

$$
\begin{aligned}
J_{k}\left(\phi^{(k)}, \psi^{(k)}\right)= & J_{k}\left(\phi^{(k)}, \psi^{(k)}\right)-\frac{1}{4} I_{k}\left(\phi^{(k)}, \psi^{(k)}\right) \\
= & \frac{1}{4}\left(\left(L_{1}-\omega_{1}\right) \phi^{(k)}, \phi^{(k)}\right)_{k} \\
& +\frac{1}{4}\left(\left(L_{2}-\omega_{2}\right) \psi^{(k)}, \psi^{(k)}\right)_{k} \\
\geq & \frac{\zeta}{4}\left(\left\|\phi^{(k)}\right\|_{k}^{2}+\left\|\psi^{(k)}\right\|_{k}^{2}\right) .
\end{aligned}
$$

This implies that

$$
\left\|\phi^{(k)}\right\|_{k}^{2}+\left\|\psi^{(k)}\right\|_{k}^{2} \leq \frac{4 \varrho}{\zeta} .
$$

The proof is completed.

Lemma 8. Assume that $\lambda_{1 *}>0$ and $\lambda_{2 *}>0$ hold. Let $\left(\phi^{(k)}, \psi^{(k)}\right)$ be a $2 k T$-periodic ground state solution, that is, a solution of (40). Then there exist positive constants $\mu$ and $\nu$ such that

$$
\mu \leq \max \left\{\left\|\phi^{(k)}\right\|_{k \infty},\left\|\psi^{(k)}\right\|_{k \infty}\right\} \leq \nu
$$

Proof. By Lemmas 4 and 6 , we know that $\left(\phi^{(k)}, \psi^{(k)}\right)$ is a nontrivial critical point of $J_{k}$. Therefore, we have

$$
\begin{aligned}
& \left(L_{1} \phi^{(k)}\right)_{n}-\omega_{1} \phi_{n}^{(k)}=a_{1}\left|\phi_{n}^{(k)}\right|^{2} \phi_{n}^{(k)}+a_{3}\left|\psi_{n}^{(k)}\right|^{2} \phi_{n}^{(k)}, \\
& \left(L_{2} \psi^{(k)}\right)_{n}-\omega_{2} \psi_{n}^{(k)}=a_{2}\left|\psi_{n}^{(k)}\right|^{2} \psi_{n}^{(k)}+a_{3}\left|\phi_{n}^{(k)}\right|^{2} \psi_{n}^{(k)} .
\end{aligned}
$$

Let $m_{i} \in \mathbb{Z}$ with $-k T \leq m_{i} \leq k T-1, i=1,2$ such that $\left\|\phi^{(k)}\right\|_{k \infty}=\left|\phi_{m_{1}}^{(k)}\right|$ and $\left\|\psi^{(k)}\right\|_{k \infty}=\left|\psi_{m_{2}}^{(k)}\right|$. By the fact that

$$
\begin{aligned}
& \left(L_{1} \phi^{(k)}\right)_{m_{1}}-\omega_{1} \phi_{m_{1}}^{(k)}=a_{1}\left|\phi_{m_{1}}^{(k)}\right|^{2} \phi_{m_{1}}^{(k)}+a_{3}\left|\psi_{m_{1}}^{(k)}\right|^{2} \phi_{m_{1}}^{(k)}, \\
& \left(L_{2} \psi^{(k)}\right)_{m_{2}}-\omega_{2} \psi_{m_{2}}^{(k)}=a_{2}\left|\psi_{m_{2}}^{(k)}\right|^{2} \psi_{m_{2}}^{(k)}+a_{3}\left|\phi_{m_{2}}^{(k)}\right|^{2} \psi_{m_{2}}^{(k)},
\end{aligned}
$$

we get

$$
\begin{aligned}
& \left|\left(L_{1}-\omega_{1}\right) \phi_{m_{1}}^{(k)}\right|=a_{1}\left\|\phi^{(k)}\right\|_{k \infty}^{3}+a_{3}\left|\psi_{m_{1}}^{(k)}\right|^{2}\left\|\phi^{(k)}\right\|_{k \infty}, \\
& \left|\left(L_{2}-\omega_{2}\right) \psi_{m_{2}}^{(k)}\right|=a_{2}\left\|\psi^{(k)}\right\|_{k \infty}^{3}+a_{3}\left|\phi_{m_{2}}^{(k)}\right|^{2}\left\|\psi^{(k)}\right\|_{k \infty} .
\end{aligned}
$$

If one of the components of $\left(\phi^{(k)}, \psi^{(k)}\right)$, say $\psi^{(k)}$, is equal to 0 , then $\phi^{(k)} \neq 0$. Thus, by (54), we obtain

$$
\begin{aligned}
& \lambda_{1 *} \leq a_{1}\left\|\phi^{(k)}\right\|_{k \infty}^{2}, \\
& \lambda_{1}^{*} \geq a_{1}\left\|\phi^{(k)}\right\|_{k \infty}^{2} .
\end{aligned}
$$

By (56), we get

$$
\sqrt{\frac{\lambda_{1 *}}{a_{1}}} \leq\left\|\phi^{(k)}\right\|_{k \infty} \leq \sqrt{\frac{\lambda_{1}^{*}}{a_{1}}}
$$


Similarly, if $\psi^{(k)} \neq 0$, then we have

$$
\sqrt{\frac{\lambda_{2 *}}{a_{2}}} \leq\left\|\psi^{(k)}\right\|_{k \infty} \leq \sqrt{\frac{\lambda_{2}^{*}}{a_{2}}} .
$$

If $\phi^{(k)} \neq 0$ and $\psi^{(k)} \neq 0$, then, by (54) and (55), we obtain

$$
\begin{gathered}
\lambda_{1 *} \leq a_{1}\left\|\phi^{(k)}\right\|_{k \infty}^{2}+a_{3}\left\|\psi^{(k)}\right\|_{k \infty}^{2}, \\
\lambda_{1}^{*} \geq a_{1}\left\|\phi^{(k)}\right\|_{k \infty}^{2}, \\
\lambda_{2 *} \leq a_{3}\left\|\phi^{(k)}\right\|_{k \infty}^{2}+a_{2}\left\|\psi^{(k)}\right\|_{k \infty}^{2}, \\
\lambda_{2}^{*} \geq a_{2}\left\|\psi^{(k)}\right\|_{k \infty}^{2} .
\end{gathered}
$$

By (59), we get

$$
\begin{aligned}
& \max \left\{\sqrt{\frac{\lambda_{1 *}}{a_{1}+a_{3}}}, \sqrt{\frac{\lambda_{2 *}}{a_{2}+a_{3}}}\right\} \\
& \leq \max \left\{\left\|\phi^{(k)}\right\|_{k \infty},\left\|\psi^{(k)}\right\|_{k \infty}\right\} \leq \max \left\{\sqrt{\frac{\lambda_{1}^{*}}{a_{1}}}, \sqrt{\frac{\lambda_{2}^{*}}{a_{2}}}\right\} .
\end{aligned}
$$

Let

$$
\begin{gathered}
\mu=\min \left\{\sqrt{\frac{\lambda_{1 *}}{a_{1}}}, \sqrt{\frac{\lambda_{2 *}}{a_{2}}}, \max \left\{\sqrt{\frac{\lambda_{1 *}}{a_{1}+a_{3}}}, \sqrt{\frac{\lambda_{2 *}}{a_{2}+a_{3}}}\right\}\right\}, \\
v=\max \left\{\sqrt{\frac{\lambda_{1}^{*}}{a_{1}}}, \sqrt{\frac{\lambda_{2}^{*}}{a_{2}}}\right\} .
\end{gathered}
$$

Then, by (57), (58), and (60), we get (51). The proof is completed.

Now we are ready to state our main results.

Theorem 9. Assume that $\omega_{1}<\theta_{11}$ and $\omega_{2}<\theta_{12}$ hold. Then for every positive integer $k$, (7) possesses a nontrivial $2 k T$-periodic ground state solution $\left(\phi^{(k)}, \psi^{(k)}\right)$ in $X_{k} \times X_{k}$. Moreover, there are other three nontrivial $2 k T$-periodic ground state solutions $\left(-\phi^{(k)}, \psi^{(k)}\right),\left(\phi^{(k)},-\psi^{(k)}\right)$, and $\left(-\phi^{(k)},-\psi^{(k)}\right)$ to $(7)$.

Proof. The proof follows easily from Lemmas 4 and 6 .

Theorem 10. Assume that $\omega_{i}<\theta_{1 i}, \lambda_{i *}>0, i=1,2$, and

$$
\begin{gathered}
a_{3}>\max \left\{\frac{\lambda+\theta_{11}-\omega_{1}}{\theta_{11}-\omega_{1}} a_{1}, \frac{\lambda+\theta_{11}-\omega_{2}}{\theta_{11}-\omega_{1}} a_{1},\right. \\
\left.\frac{\lambda+\theta_{12}-\omega_{1}}{\theta_{12}-\omega_{2}} a_{2}, \frac{\lambda+\theta_{12}-\omega_{2}}{\theta_{12}-\omega_{2}} a_{2}\right\}
\end{gathered}
$$

holds. Then system (7) has a nontrivial ground state solution $\left(\phi^{*}, \psi^{*}\right)$ in $X \times X$ with $\phi^{*} \neq 0$ and $\psi^{*} \neq 0$. Moreover, there are other three nontrivial ground state solutions $\left(-\phi^{*}, \psi^{*}\right)$, and $\left(\phi^{*},-\psi^{*}\right),\left(-\phi^{*},-\psi^{*}\right)$ to $(7)$.
Proof. Consider the sequence $\left\{\left(\phi^{(k)}, \psi^{(k)}\right)\right\}$ of $2 k T$-periodic solutions found in Lemma 6. By Lemma 8, without loss of generality, we can assume that the subsequence $\left\{\left(\phi^{\left(k_{i}\right)}, \psi^{\left(k_{i}\right)}\right)\right\}$ of $\left\{\left(\phi^{(k)}, \psi^{(k)}\right)\right\}$ satisfies $\mu \leq\left\|\phi^{\left(k_{i}\right)}\right\|_{k \infty} \leq \nu$. Thus, there exists $n_{k_{i}} \in \mathbb{Z}$ such that

$$
\left|\phi_{n_{k_{i}}}^{\left(k_{i}\right)}\right| \geq \mu
$$

By the periodicity of the coefficients in (7), we see that $\left\{\left(\phi_{n+T}^{(k)}, \psi_{n+T}^{(k)}\right)\right\}$ is also a solution to (7). Making some shifts if necessary, without loss of generality, we can assume that $0 \leq n_{k_{i}} \leq T-1$ in (63). Moreover, passing to a subsequence $\left\{\phi^{\left(k_{i_{j}}\right)}\right\}$ of $\left\{\phi^{\left(k_{i}\right)}\right\}$, we can also assume that $n_{k_{i}}=n^{*}$ and $0 \leq n^{*} \leq T-1$. It follows from (37), (50), (51), and (63) that we can choose a subsequence, still denoted by $\left\{\phi^{(k)}\right\}$ and $\left\{\psi^{(k)}\right\}$, such that $\phi_{n}^{(k)} \rightarrow \phi_{n}^{*}$ and $\psi_{n}^{(k)} \rightarrow \psi_{n}^{*}$ for all $n \in \mathbb{Z}$. Notice that $\left|\phi_{n^{*}}^{*}\right| \geq \mu$. Then $\left(\phi^{*}, \psi^{*}\right) \in X \times X$ with $\left(\phi^{*}, \psi^{*}\right) \neq(0,0)$. Furthermore, (7) possesses pointwise limits and hence $\left(\phi^{*}, \psi^{*}\right)$ is a nontrivial solution to (7). By the way, $\left(-\phi^{*}, \psi^{*}\right),\left(\phi^{*},-\psi^{*}\right)$, and $\left(-\phi^{*},-\psi^{*}\right)$ are nontrivial solutions to (7). Since $J\left(\phi^{*}, \psi^{*}\right)=J\left(-\phi^{*}, \psi^{*}\right)=J\left(\phi^{*},-\psi^{*}\right)=$ $J\left(-\phi^{*},-\psi^{*}\right)$, if $\left(\phi^{*}, \psi^{*}\right)$ is a ground state solution, then $\left(-\phi^{*}, \psi^{*}\right),\left(\phi^{*},-\psi^{*}\right)$, and $\left(-\phi^{*},-\psi^{*}\right)$ are the ground state solutions.

Now we prove that the solution $\left(\phi^{*}, \psi^{*}\right)$ is a ground state solution. By Lemma 5, we have to show that $J\left(\phi^{*}, \psi^{*}\right)=m$. Actually, we have proven that, for every sequence $k_{j} \rightarrow \infty$, passing to a subsequence still denoted by $k_{j}$ and making appropriate shifts, we can suppose that $\phi_{n}^{\left(k_{j}\right)} \rightarrow \phi_{n}^{*}$ and $\psi_{n}^{\left(k_{j}\right)} \rightarrow \psi_{n}^{*}$ pointwise, where $\left(\phi^{*}, \psi^{*}\right) \in X \times X$ is a nontrivial solution. For any positive integer $K$, we have

$$
\begin{aligned}
& \liminf _{j \rightarrow \infty} J_{k_{j}}\left(\phi^{\left(k_{j}\right)}, \psi^{\left(k_{j}\right)}\right) \\
& =\liminf _{j \rightarrow \infty} \frac{1}{4} \sum_{n=-k_{j} T}^{k_{j} T-1}\left(a_{1}\left(\phi_{n}^{\left(k_{j}\right)}\right)^{4}+a_{2}\left(\psi_{n}^{\left(k_{j}\right)}\right)^{4}\right. \\
& \left.+2 a_{3}\left(\phi_{n}^{\left(k_{j}\right)}\right)^{2}\left(\psi_{n}^{\left(k_{j}\right)}\right)^{2}\right) \\
& \geq \liminf _{j \rightarrow \infty} \frac{1}{4} \sum_{n=-K T}^{K T-1}\left(a_{1}\left(\phi_{n}^{\left(k_{j}\right)}\right)^{4}+a_{2}\left(\psi_{n}^{\left(k_{j}\right)}\right)^{4}\right. \\
& \left.+2 a_{3}\left(\phi_{n}^{\left(k_{j}\right)}\right)^{2}\left(\psi_{n}^{\left(k_{j}\right)}\right)^{2}\right) \\
& =\frac{1}{4} \sum_{n=-K T}^{K T-1}\left(a_{1}\left(\phi_{n}^{*}\right)^{4}+a_{2}\left(\psi_{n}^{*}\right)^{4}+2 a_{3}\left(\phi_{n}^{*}\right)^{2}\left(\psi_{n}^{*}\right)^{2}\right) .
\end{aligned}
$$

Let $K \rightarrow \infty$; we obtain that

$$
\liminf _{j \rightarrow \infty} J_{k_{j}}\left(\phi^{\left(k_{j}\right)}, \psi^{\left(k_{j}\right)}\right) \geq J\left(\phi^{(*)}, \psi^{(*)}\right) \geq m,
$$

and, hence,

$$
\liminf _{k \rightarrow \infty} m_{k} \geq m
$$


Now we prove that

$$
\limsup _{k \rightarrow \infty} m_{k} \leq m
$$

By Lemma 7, we know that the sequence $\left\{m_{k}\right\}$ is bounded above and below away from zero. We extract a subsequence, still denoted by $\left\{m_{k}\right\}$, and hence we prove that

$$
\lim _{k \rightarrow \infty} m_{k} \leq m
$$

Given $\tau>0$, let $(\widehat{\phi}, \widehat{\psi}) \in N$ be such that

$$
\begin{aligned}
& J(\widehat{\phi}, \widehat{\psi}) \\
& \quad=\frac{1}{4} \sum_{n \in \mathbb{Z}}\left(a_{1}\left(\widehat{\phi}_{n}\right)^{4}+a_{2}\left(\widehat{\psi}_{n}\right)^{4}+2 a_{3}\left(\widehat{\phi}_{n}\right)^{2}\left(\widehat{\psi}_{n}\right)^{2}\right)<m+\tau .
\end{aligned}
$$

Choose $t_{1}>1$ sufficiently close to 1 such that

$$
\frac{1}{4} \sum_{n \in \mathbb{Z}}\left(a_{1}\left(t_{1} \widehat{\phi}_{n}\right)^{4}+a_{2}\left(t_{1} \widehat{\psi}_{n}\right)^{4}+2 a_{3}\left(t_{1} \widehat{\phi}_{n}\right)^{2}\left(t_{1} \widehat{\psi}_{n}\right)^{2}\right)<m+\tau .
$$

We also have that $I\left(t_{1} \widehat{\phi}, t_{1} \widehat{\psi}\right)<0$. By density argument, we can find a finitely supported sequence $(\widetilde{\phi}, \widetilde{\psi})$ sufficiently close to $\left(t_{1} \widehat{\phi}, t_{1} \widehat{\psi}\right)$ in $X$ such that $I(\widetilde{\phi}, \widetilde{\psi})<0$ and

$$
\frac{1}{4} \sum_{n \in \mathbb{Z}}\left(a_{1}\left(\widetilde{\phi}_{n}\right)^{4}+a_{2}\left(\widetilde{\psi}_{n}\right)^{4}+2 a_{3}\left(\widetilde{\phi}_{n}\right)^{2}\left(\widetilde{\psi}_{n}\right)^{2}\right)<m+\tau .
$$

Then there exists $t_{2} \in(0,1)$ such that $I\left(t_{2} \widetilde{\phi}, t_{2} \widetilde{\psi}\right)=0$ and

$$
\begin{aligned}
& J\left(t_{2} \widetilde{\phi}, t_{2} \widetilde{\psi}\right) \\
& =\frac{1}{4} \sum_{n \in \mathbb{Z}}\left(a_{1}\left(t_{2} \widetilde{\phi}_{n}\right)^{4}+a_{2}\left(t_{2} \widetilde{\psi}_{n}\right)^{4}+2 a_{3}\left(t_{2} \widetilde{\phi}_{n}\right)^{2}\left(t_{2} \widetilde{\psi}_{n}\right)^{2}\right) \\
& \quad<\frac{1}{4} \sum_{n \in \mathbb{Z}}\left(a_{1}\left(\widetilde{\phi}_{n}\right)^{4}+a_{2}\left(\widetilde{\psi}_{n}\right)^{4}+2 a_{3}\left(\widetilde{\phi}_{n}\right)^{2}\left(\widetilde{\psi}_{n}\right)^{2}\right)<m+\tau .
\end{aligned}
$$

Let $\left(\widetilde{\phi}^{(k)}, \widetilde{\psi}^{(k)}\right) \in X_{k} \times X_{k}$ be such that $\widetilde{\phi}_{n}^{(k)}=\widetilde{\phi}_{n}$ and $\widetilde{\psi}_{n}^{(k)}=\widetilde{\psi}_{n}$ if $n \in \mathbb{Z} \cap[-k T, k T-1]$. If $k$ is large enough, then $I_{k}\left(t_{2} \widetilde{\phi}^{(k)}, t_{2} \widetilde{\psi}^{(k)}\right)=I\left(t_{2} \widetilde{\phi}, t_{2} \widetilde{\psi}\right)=0$ and

$$
J_{k}\left(t_{2} \widetilde{\phi}^{(k)}, t_{2} \widetilde{\psi}^{(k)}\right)=J\left(t_{2} \widetilde{\phi}, t_{2} \widetilde{\psi}\right)<m+\tau \text {. }
$$

This implies (68). Hence, by (66) and (68), we have $J\left(\phi^{*}\right.$, $\left.\psi^{*}\right)=m$.

Finally, we will show that $\phi^{*} \neq 0$ and $\psi^{*} \neq 0$. From the above arguments, system (7) has a nontrivial ground state solution $\left(\phi^{*}, \psi^{*}\right)$ in $X \times X$. If one of the components of $\left(\phi^{*}, \psi^{*}\right)$, say $\psi^{*}$, is equal to 0 , then $\phi^{*} \neq 0$ (the proof for the other case is similar). For $\epsilon$ small enough, we consider $\left(\phi^{*}, \epsilon \phi^{*}\right) \in(X-\{0\}) \times(X-\{0\})$. By a similar argument to the proof of Lemma 1, we know that there exists $t^{*}$ such that $I\left(t^{*} \phi^{*}, t^{*} \epsilon \phi^{*}\right)=0$; that is $\left(t^{*} \phi^{*}, t^{*} \epsilon \phi^{*}\right) \in N$. By $I\left(t^{*} \phi^{*}, t^{*} \epsilon \phi^{*}\right)=0$, we have $\left(t^{*}\right)^{2}=$ $H_{1}\left(\phi^{*}, \epsilon \phi^{*}\right) / H_{2}\left(\phi^{*}, \epsilon \phi^{*}\right)$, where

$$
\begin{gathered}
H_{1}(\phi, \psi)=\left(\left(L_{1}-\omega_{1}\right) \phi, \phi\right)+\left(\left(L_{2}-\omega_{2}\right) \psi, \psi\right), \\
H_{2}(\phi, \psi)=\sum_{n \in \mathbb{Z}}\left(a_{1}\left(\phi_{n}\right)^{4}+a_{2}\left(\psi_{n}\right)^{4}+2 a_{3}\left(\phi_{n}\right)^{2}\left(\psi_{n}\right)^{2}\right) .
\end{gathered}
$$

Moreover, $J\left(t^{*} \phi^{*}, t^{*} \epsilon \phi^{*}\right)=H_{1}^{2}\left(\phi^{*}, \epsilon \phi^{*}\right) /\left(4 H_{2}\left(\phi^{*}, \epsilon \phi^{*}\right)\right)$. Notice that $J\left(\phi^{*}, 0\right)=\inf _{(\phi, \psi) \in N} J(\phi, \psi), J\left(\phi^{*}, 0\right)=\left(a_{1} / 4\right)$ $\sum_{n \in \mathbb{Z}}\left(\phi_{n}^{*}\right)^{4}$, and

$$
\begin{aligned}
H_{2}\left(\phi^{*}, 0\right) & =H_{1}\left(\phi^{*}, 0\right)=\left(\left(L_{1}-\omega_{1}\right) \phi^{*}, \phi^{*}\right) \\
& \geq\left(\theta_{11}-\omega_{1}\right)\left\|\phi^{*}\right\|^{2} .
\end{aligned}
$$

For the sake of simplicity, we let

$$
B=\sum_{n \in \mathbb{Z}}\left(\phi_{n}^{*}\right)^{4}, \quad D=\left(\left(L_{2}-\omega_{2}\right) \phi^{*}, \phi^{*}\right) .
$$

If $\omega_{1} \leq \omega_{2}$, then, by (28) and (75),

$$
\begin{aligned}
D= & \left(\left(L_{2}-\omega_{2}\right) \phi^{*}, \phi^{*}\right) \\
= & \left(\left(L_{1}-\omega_{1}\right) \phi^{*}, \phi^{*}\right)+\left(\left(L_{2}-L_{1}\right) \phi^{*}, \phi^{*}\right) \\
& +\left(\left(\omega_{1}-\omega_{2}\right) \phi^{*}, \phi^{*}\right) \\
\leq & a_{1} B+\lambda\left\|\phi^{*}\right\|^{2} \leq \frac{\lambda+\theta_{11}-\omega_{1}}{\theta_{11}-\omega_{1}} a_{1} B .
\end{aligned}
$$

This, combined with $a_{3}>\left(\left(\lambda+\theta_{11}-\omega_{1}\right) /\left(\theta_{11}-\omega_{1}\right)\right) a_{1}$ and (77) yields $a_{1} B D<a_{1} a_{3} B^{2}$. If $\omega_{2}<\omega_{1}$, then, by (28) and (75),

$$
\begin{aligned}
D= & \left(\left(L_{2}-\omega_{2}\right) \phi^{*}, \phi^{*}\right) \\
= & \left(\left(L_{1}-\omega_{1}\right) \phi^{*}, \phi^{*}\right)+\left(\left(L_{2}-L_{1}\right) \phi^{*}, \phi^{*}\right) \\
& +\left(\left(\omega_{1}-\omega_{2}\right) \phi^{*}, \phi^{*}\right) \\
= & a_{1} B+\lambda\left\|\phi^{*}\right\|^{2}+\left(\omega_{1}-\omega_{2}\right)\left\|\phi^{*}\right\|^{2} \\
\leq & a_{1} B+\frac{\lambda+\omega_{1}-\omega_{2}}{\theta_{11}-\omega_{1}} a_{1} B=\frac{\lambda+\theta_{11}-\omega_{2}}{\theta_{11}-\omega_{1}} a_{1} B .
\end{aligned}
$$

This, together with $a_{3}>\left(\left(\lambda+\theta_{11}-\omega_{2}\right) /\left(\theta_{11}-\omega_{1}\right)\right) a_{1}$ and (78) gives $a_{1} B D<a_{1} a_{3} B^{2}$. Thus, if $a_{3}>\max \left\{\left(\left(\lambda+\theta_{11}-\omega_{1}\right) /\right.\right.$ $\left.\left.\left(\theta_{11}-\omega_{1}\right)\right) a_{1},\left(\left(\lambda+\theta_{11}-\omega_{2}\right) /\left(\theta_{11}-\omega_{1}\right)\right) a_{1}\right\}$, then we have $a_{1} B D<a_{1} a_{3} B^{2}$. For $\epsilon$ small enough, we have

$$
\begin{aligned}
H_{1}^{2}\left(\phi^{*}, \epsilon \phi^{*}\right) & =\left(\left(\left(L_{1}-\omega_{1}\right) \phi^{*}, \phi^{*}\right)+\left(\left(L_{2}-\omega_{2}\right) \epsilon \phi^{*}, \epsilon \phi^{*}\right)\right)^{2} \\
& =\left(a_{1} B+\epsilon^{2} D\right)^{2}=a_{1}^{2} B^{2}+2 a_{1} B D \epsilon^{2}+D^{2} \epsilon^{4} \\
& <a_{1}^{2} B^{2}+2 a_{1} a_{3} B^{2} \epsilon^{2}+a_{1} a_{2} B^{2} \epsilon^{4} \\
& =a_{1} B\left(a_{1} B+a_{2} B \epsilon^{4}+2 a_{3} B \epsilon^{2}\right) \\
& =H_{2}\left(\phi^{*}, 0\right) H_{2}\left(\phi^{*}, \epsilon \phi^{*}\right) .
\end{aligned}
$$


Hence, by (79), we have

$$
\begin{aligned}
J\left(t^{*} \phi^{*}, t^{*} \epsilon \phi^{*}\right) & =\frac{H_{1}^{2}\left(\phi^{*}, \epsilon \phi^{*}\right)}{4 H_{2}\left(\phi^{*}, \epsilon \phi^{*}\right)}<\frac{1}{4} H_{2}\left(\phi^{*}, 0\right) \\
& =J\left(\phi^{*}, 0\right)=\inf _{(\phi, \psi) \in N} J(\phi, \psi) .
\end{aligned}
$$

This is a contradiction. So, $\psi^{*} \neq 0$. The proof is completed.

\section{Acknowledgments}

The authors would like to thank the anonymous referee for his/her valuable suggestions. This work is supported by Program for Changjiang Scholars and Innovative Research Team in University (no. IRT1226), the National Natural Science Foundation of China (no. 11171078), the Specialized Fund for the Doctoral Program of Higher Education of China (no. 20114410110002), and SRF of Guangzhou Education Bureau (no. 10A012).

\section{References}

[1] M. Caliari and M. Squassina, "Location and phase segregation of ground and excited states for 2D Gross-Pitaevskii systems," Dynamics of Partial Differential Equations, vol. 5, no. 2, pp. 117137, 2008.

[2] A. Ambrosetti and E. Colorado, "Standing waves of some coupled nonlinear Schrödinger equations," Journal of the London Mathematical Society, vol. 75, no. 1, pp. 67-82, 2007.

[3] C. J. Myatt, E. A. Burt, R. W. Ghrist, E. A. Cornell, and C. E. Wieman, "Production of two overlapping Bose-Einstein condensates by sympathetic cooling," Physical Review Letters, vol. 78, no. 4, pp. 586-589, 1997.

[4] R. S. Mackay and S. Aubry, "Proof of existence of breathers for time-reversible or Hamiltonian networks of weakly coupled oscillators," Nonlinearity, vol. 7, no. 6, pp. 1623-1643, 1994.

[5] S. Aubry, "Breathers in nonlinear lattices: existence, linear stability and quantization," Physica D, vol. 103, no. 1-4, pp. 201-250, 1997.

[6] P. G. Kevrekidis, The Discrete Nonlinear Schrödinger Equation, Springer, 2009

[7] D. E. Pelinovsky and V. M. Rothos, "Bifurcations of travelling wave solutions in the discrete NLS equations," Physica D, vol. 202, no. 1-2, pp. 16-36, 2005.

[8] A. Pankov and V. Rothos, "Periodic and decaying solutions in discrete nonlinear Schrödinger with saturable nonlinearity," Proceedings of The Royal Society of London A, vol. 464, no. 2100, pp. 3219-3236, 2008.

[9] H. Shi and H. Zhang, "Existence of gap solitons in a periodic discrete nonlinear Schrödinger equations," Journal of Mathematical Analysis and Applications, vol. 361, no. 2, pp. 411-419, 2010.

[10] G. Zhang and F. Liu, "Existence of breather solutions of the DNLS equations with unbounded potentials," Nonlinear Analysis: Theory, Methods \& Applications, vol. 71, no. 12, pp. e786e792, 2009.

[11] Z. Zhou and J. Yu, "On the existence of homoclinic solutions of a class of discrete nonlinear periodic systems," Journal of Differential Equations, vol. 249, no. 5, pp. 1199-1212, 2010.
[12] Z. Zhou, J. Yu, and Y. Chen, "On the existence of gap solitons in a periodic discrete nonlinear Schrödinger equation with saturable nonlinearity," Nonlinearity, vol. 23, no. 7, pp. 17271740, 2010.

[13] X. Deng, H. Shi, and X. Xie, "Periodic solutions of second order discrete Hamiltonian systems with potential indefinite in sign," Applied Mathematics and Computation, vol. 218, no. 1, pp. 148156, 2011.

[14] X. Zhang and X. Tang, "Subharmonic solutions for a class of non-quadratic second order Hamiltonian systems," Nonlinear Analysis: Real World Applications, vol. 13, no. 1, pp. 113-130, 2012.

[15] P. Chen, X. Tang, and R. P. Agarwal, "Fast homoclinic solutions for a class of damped vibration problems," Applied Mathematics and Computation, vol. 219, no. 11, pp. 6053-6065, 2013.

[16] J. Belmonte-Beitia, V. M. Pérez-García, and P. J. Torres, "Solitary waves for linearly coupled nonlinear Schrödinger equations with inhomogeneous coefficients," Journal of Nonlinear Science, vol. 19, no. 4, pp. 437-451, 2009.

[17] G. Teschl, Jacobi Operators and Completely Integrable Nonlinear Lattices, vol. 72 of Mathematical Surveys and Monographs, American Mathematical Society, Providence, RI, USA, 2000. 


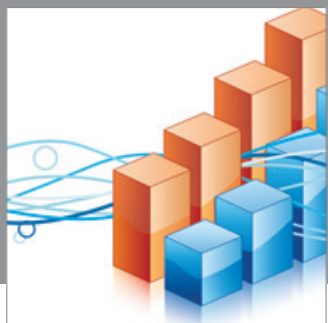

Advances in

Operations Research

mansans

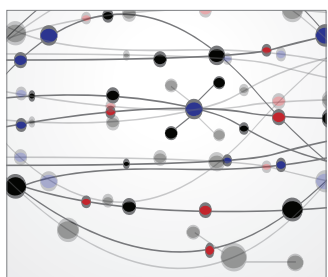

The Scientific World Journal
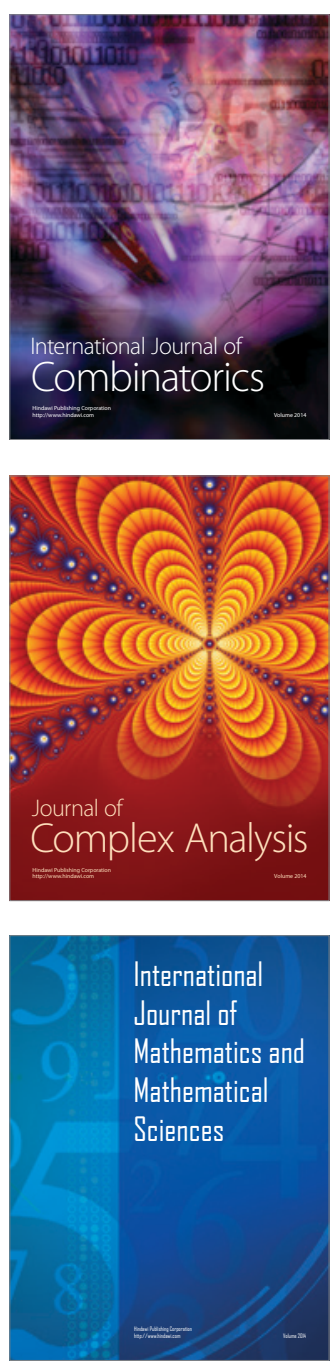
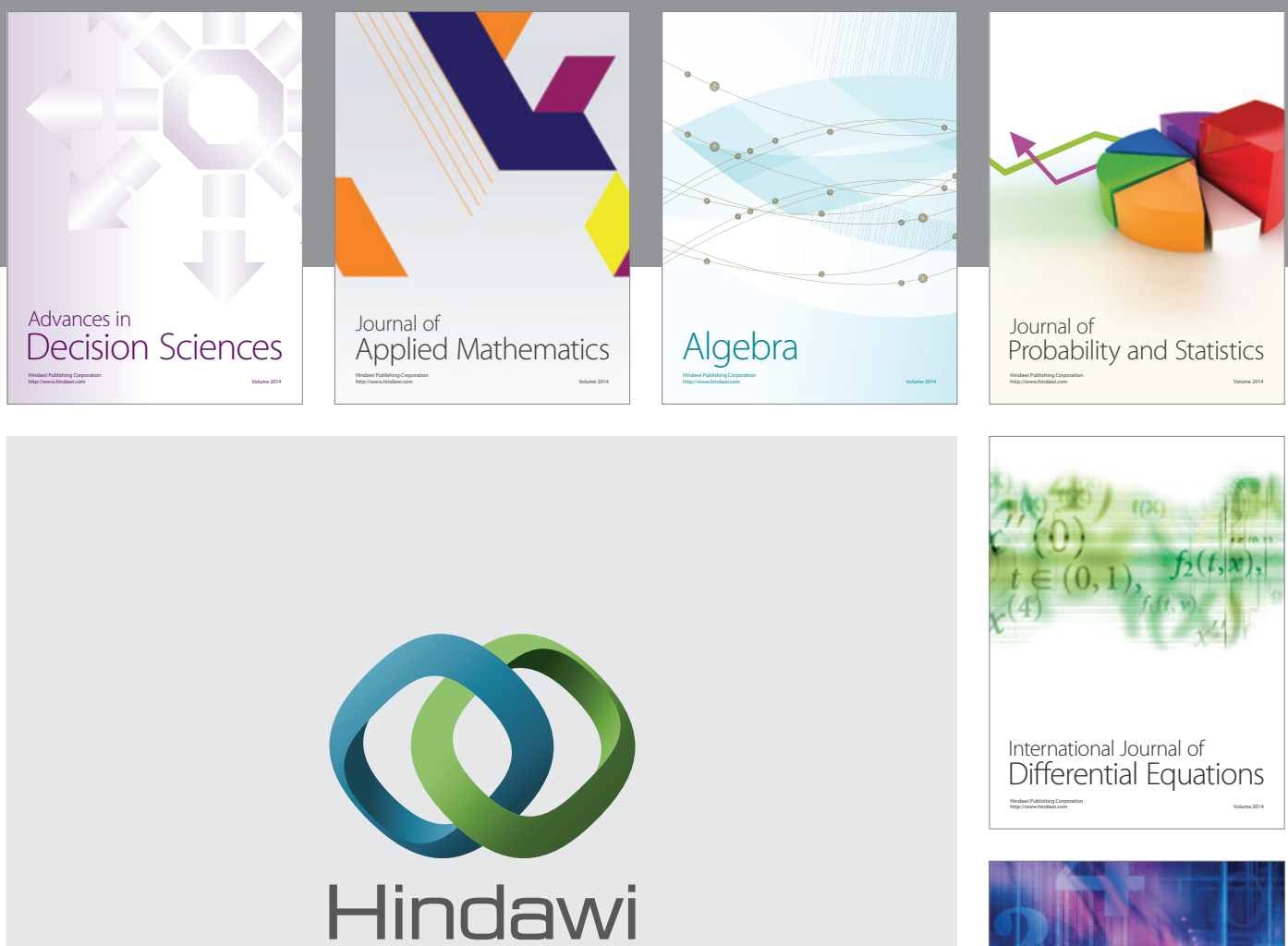

Submit your manuscripts at http://www.hindawi.com
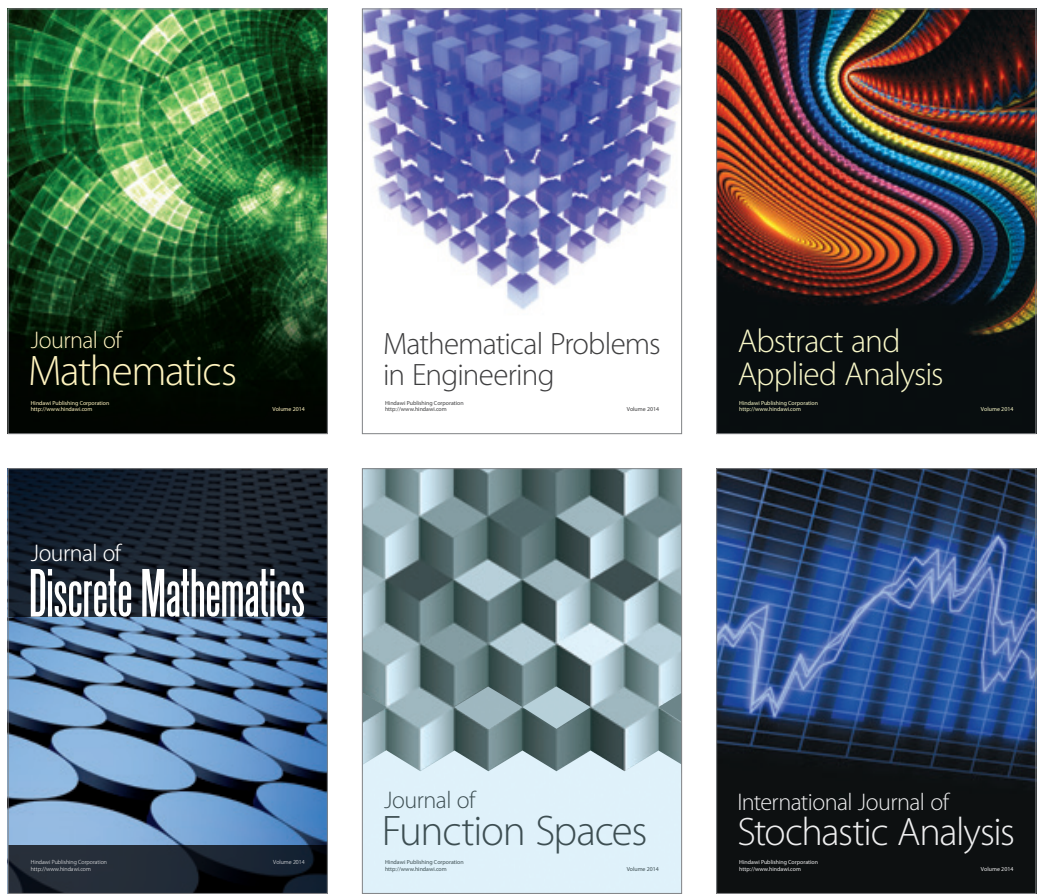

Journal of

Function Spaces

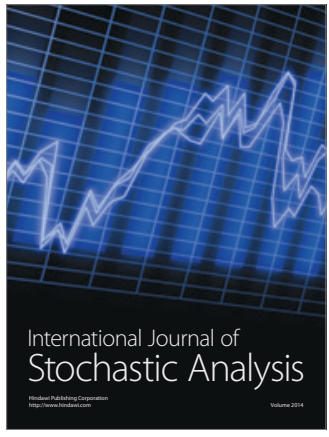

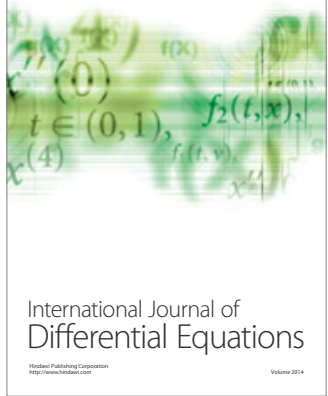
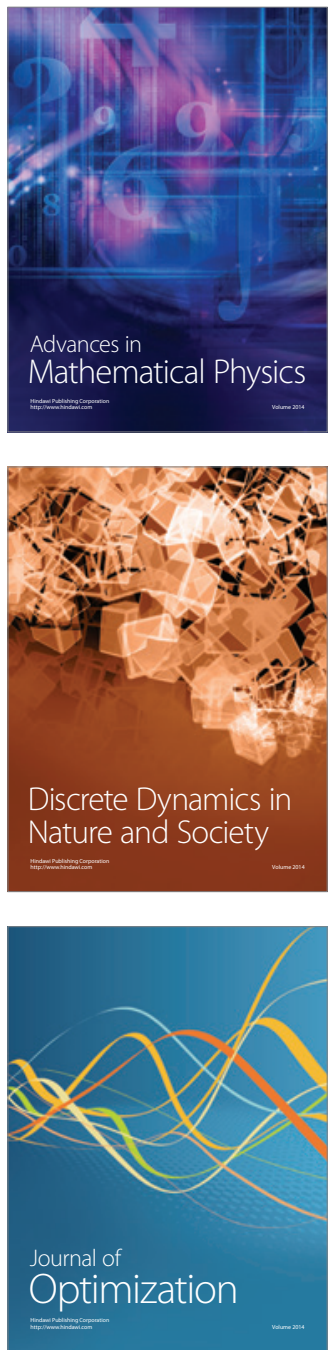